\title{
The multiple-scattering series in pion-deuteron scattering and the nucleon-nucleon potential: perspectives from effective field theory
}

\author{
V. Baru ${ }^{1,2}$, E. Epelbaum ${ }^{1}$, C. Hanhart ${ }^{3,4}$, M. Hoferichter ${ }^{5,6}$, A. E. Kudryavtsev ${ }^{2}$, and D. R. Phillips ${ }^{6}$ \\ 1 Institut für Theoretische Physik II, Ruhr-Universität Bochum, D-44780 Bochum, Germany \\ 2 Institute for Theoretical and Experimental Physics, B. Cheremushinskaya 25, 117218 Moscow, Russia \\ 3 Institut für Kernphysik and Jülich Center for Hadron Physics, Forschungszentrum Jülich, D-52425 Jülich, Germany \\ ${ }^{4}$ Institute for Advanced Simulation, Forschungszentrum Jülich, D-52425 Jülich, Germany \\ 5 Helmholtz-Institut für Strahlen- und Kernphysik and Bethe Center for Theoretical Physics, Universität Bonn, D-53115 Bonn, Germany \\ ${ }^{6}$ Institute of Nuclear and Particle Physics and Department of Physics and Astronomy, Ohio University, Athens, OH 45701, USA
}

\begin{abstract}
Important contributions to meson-nucleus scattering are produced by terms in the multiple-scattering series, which is defined as the sum of all diagrams where the meson scatters back and forth between a pair of static nucleons before leaving the nucleus. In particular, the sum of this series is needed for an accurate description of kaondeuteron scattering, and appears as part of the nucleon-nucleon potential. In this article we present some effective-fieldtheory (EFT)-based insights into this series in the case of two-nucleon systems. In particular, we discuss the fact that, if meson-nucleon scattering is approximated by the scattering-length term, individual terms of the series are divergent, and enhanced with respect to the straightforward expectation from chiral perturbation theory $(\chi \mathrm{PT})$. This apparently indicates the presence of similarly enhanced counterterms. However, we show that when the series is resummed the divergences cancel, such that no additional information on short-range interactions is needed to obtain predictions for observables after resummation. We discuss the conditions under which this resummation is justified. We show that the same issues arise in the $N N$ potential, where the resummed series produces poles whose appearance indicates the breakdown scale of the $\chi \mathrm{PT}$ expansion for that quantity. This demonstrates unequivocally that $\chi \mathrm{PT}$ cannot be applied to compute $V(r)$ for distances smaller than $r \sim 1 \mathrm{fm}$ at least in the theory without explicit Delta(1232) degrees of freedom. We briefly discuss whether this bound can be lowered if the Delta resonance is included in the EFT as an explicit degree of freedom.
\end{abstract}

PACS. 13.75.Gx Pion-baryon interactions - 12.39.Fe Chiral Lagrangians - 36.10.Gv Mesonic, hyperonic and antiprotonic atoms and molecules

\section{Introduction}

The multiple-scattering series (MSS) has played a prominent role in the study of meson-nucleus interactions. Explicit expressions for the MSS have been known for a long time: first derived in 1949 by Foldy in a different context [1], it was applied to $\pi d$ scattering as early as 1953 by Brückner [2]. The first diagrammatic representation was given in Ref. [3] in 1972. More recently, the terms of the MSS have been shown to have a special status within an effective-field-theory treatment for $\pi$ nucleus scattering. The first EFT calculation of $\pi d$ scattering, performed by Weinberg [4], classified the different contributions to the pion-deuteron scattering length, $a_{\pi d}$, according to their $\chi \mathrm{PT}$ order. Of the three three-body diagrams at leading order, by far the largest one is due to the second term in the $\pi d$ MSS, the so-called double-scattering term (see first diagram in Fig. (1). Weinberg's calculation has been refined in the twenty years since (see, e.g., Refs. [5, 6,7, 8, 9, 10, 11, 12]). In Ref. [6] it was observed that the triple-scattering term (i.e. the third term in the MSS) is significantly enhanced compared to what one would expect based on Weinberg's original dimensionalanalysis argument. In contrast to what was proposed in Ref. [6], it was shown in Ref. [10] that the triple-scattering term is enhanced by a factor of $\pi^{2}$ compared to its naive $\chi \mathrm{PT}$ order because of its special topology. The large contribution of the first diagram in Fig. 1 to $a_{\pi d}$, together with the enhanced contribution of the second one, raises the question of whether all diagrams in the MSS are enhanced as compared to other $\chi \mathrm{PT}$ graphs in one way or another.

This question is of considerable contemporary import, as data on mesonic atoms have, in recent years, become a prime source of experimental information on strong meson-nucleon scattering lengths. In particular, the pion-nucleon scattering lengths were extracted from a combined analysis of pionic hydrogen [13] and pionic deuterium [14] data with unprecedented accuracy in Refs. [11,12]. Such an analysis calls for rigorous control over higher-order $\chi \mathrm{PT}$ corrections to pionnucleus scattering, potentially the most prominent of which are 
the higher-order terms of the MSS. Due to the smallness of the $\pi N$ scattering lengths, terms beyond triple scattering in the MSS give small contributions to $a_{\pi d}$. However, such a suppression does not show up for $K d$ scattering due to the relatively large $K N$ scattering lengths of the order of $1 \mathrm{fm}$. In this case the non-perturbative resummation of all terms in the MSS is required [15, 16]1]. The goal of this study is to examine the consequences of enhancements of MSS terms for the $\chi \mathrm{PT}$ counting, and the grounds for such a resummation.

Our main result is that care is required when expanding the MSS in a diagrammatic fashion. In particular, a perturbative treatment of the series necessitates the introduction of enhanced counterterms if well-defined expressions are to be obtained. This is because the integrals appearing in the diagrammatic expansion are individually divergent, starting from the quadruple-scattering term in the series (third diagram in Fig.(1). Nevertheless, we show that, under particular circumstances, all those divergences cancel upon resummation. Based on this observation we are also able to present a closed expression for the MSS in momentum space.

The pertinent terms in the MSS also appear as sub-graphs within the $\chi \mathrm{PT}$ contributions to the $N N$ potential. (It was pointed out long ago that the sum of all two-particle irreducible $\pi N N \rightarrow \pi N N$ graphs itself appears in $V_{N N}$ [18, 19, 20, 21, 22].) Here we show that "triangle graphs" in the $N N$ potential are enhanced by factors of $\pi$ (not $\pi^{2}$ ) providing a special status to the diagrams of the MSS - and the physics insights derived therefrom - in that problem, too. We find that the MSS contributions to $V_{N N}$ can be (partially) resummed. The effect of higher-order MSS terms is minimal for $r \geq 1 \mathrm{fm}$, but, for distances $r<1 \mathrm{fm}$, they produce unphysical poles in $V_{N N}(r)$. This leads us to suggest that their appearance is associated with the breakdown of the $\chi \mathrm{PT}$ expansion for the $N N$ potential at these distances.

The remainder of this paper is organized as follows. In Sect. 2 we introduce the multiple-scattering series and define our conventions. In Sect. 3 we perform a perturbative evaluation of the graphs in this series and show that counterterms are needed in order to make sense of the divergent momentumspace expressions which are encountered. In Sect.4 we provide a formal argument which vitiates the need to consider these counterterms. We first regularize each term in the MSS, then resum the series, and finally remove the regulator and obtain a finite result. In Sect. 5]we discuss the limitations of this procedure. In Sect. 6 we apply our insights from the meson-nucleus case to the more complex case of the $N N$ potential. We offer our conclusions in Sect.7

\section{The multiple-scattering series}

For the sake of simplicity, we start with isoscalar mesons scattering off isoscalar nucleons. Then the MSS for meson-nucleus

\footnotetext{
${ }^{1}$ For a discussion of the role of recoil corrections potentially relevant for $K d$ scattering, see Ref. [17].
}
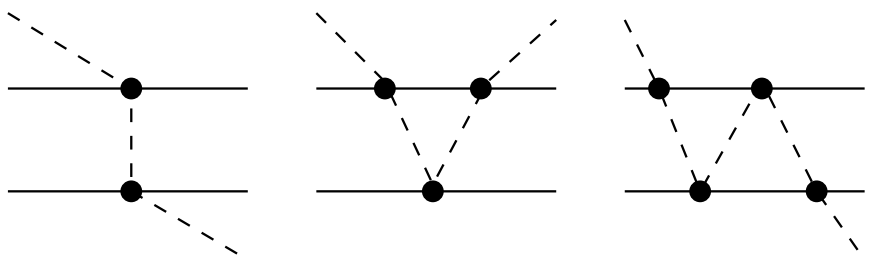

Fig. 1. Second, third, and fourth term in the MSS. Solid lines denote nucleons, dashed mesons, and solid blobs interactions amongst them.

scattering may be written as

$$
2 T=2(4 \pi)^{2}\left(A^{(1)}+A^{(2)}+A^{(3)}+A^{(4)}+\cdots\right),
$$

where the superscript indicates the number of meson-nucleon interactions appearing in the respective diagram, and a factor $2(4 \pi)^{2}$ has been pulled out for convenience. The second, third, and forth terms in the series are illustrated in Fig. 1.

The $n$th term in the series shown in Fig.11has $n-2$ loops. However, once nucleons are treated as static, the amplitude for a zero-momentum pion scattering from the $N N$ system can be computed using the integral equation

$$
\begin{aligned}
T\left(\mathbf{p}^{\prime}, \mathbf{p}\right) & =t_{\pi N}(\mathbf{0}, \mathbf{0})(2 \pi)^{3} \delta^{(3)}\left(\mathbf{p}^{\prime}-\mathbf{p}\right) \\
& +\int \frac{d^{3} p^{\prime \prime}}{(2 \pi)^{3}} t_{\pi N}\left(\mathbf{p}-\mathbf{p}^{\prime \prime}, \mathbf{0}\right) \frac{1}{\left(\mathbf{p}-\mathbf{p}^{\prime \prime}\right)^{2}} T\left(\mathbf{p}^{\prime}, \mathbf{p}^{\prime \prime}\right),
\end{aligned}
$$

where $\mathbf{p}\left(\mathbf{p}^{\prime}\right)$ is the relative momentum between the incoming (outgoing) $N N$ pair and $t_{\pi N}\left(\mathbf{q}^{\prime}, \mathbf{q}\right)$ describes a $\pi N$ interaction with final (initial) pion momentum $\mathbf{q}^{\prime}(\mathbf{q})$. The contribution to the $\pi d$ scattering length stemming from the multiple-scattering series, Eq. (2), then read 3

$$
a_{\pi d}=-\frac{1}{16 \pi^{4}} \int d^{3} p d^{3} p^{\prime} \Psi^{\dagger}\left(\mathbf{p}^{\prime}\right) T\left(\mathbf{p}^{\prime}, \mathbf{p}\right) \Psi(\mathbf{p}),
$$

where $\Psi(\mathbf{p})$ denotes the deuteron wave function normalized as

$$
\int d^{3} p \Psi^{\dagger}(\mathbf{p}) \Psi(\mathbf{p})=1
$$

If only low-momentum components of the nuclear system are being probed, we are encouraged to take

$$
t_{\pi N}(\mathbf{0}, \mathbf{0})=t_{\pi N}\left(\mathbf{p}-\mathbf{p}^{\prime \prime}, \mathbf{0}\right)=-4 \pi a,
$$

where $a$ is the $\pi N$ scattering length. (Note that we neglected the kinematical prefactors suppressed as $M_{\pi} / m_{N}$ with $m_{N}$ being the nucleon mass.) In $\chi \mathrm{PT}$ the absolute value of the isovector $\pi N$ scattering length is

$$
a=\frac{M_{\pi}}{8 \pi F_{\pi}^{2}}
$$

\footnotetext{
${ }^{2}$ The full MSS is given by $2 T$ because the pion can scatter from either nucleon to start any term in the MSS.

3 Throughout this work we use nuclear-physics conventions for the scattering lengths. Note that this differs from the conventions employed in previous studies of $\pi d$ scattering [6 10 11 12 ].
} 
a result that will be needed below for the power counting, as an example of a natural meson-nucleon scattering length, even though we mainly consider isoscalar meson-nucleon scatteringt.

\section{Perturbative evaluation}

If $t_{\pi N}$ has the form (5), the first two terms of the MSS may be written as

$$
\begin{aligned}
& A^{(1)}(\mathbf{Q})=-2 \pi^{2} a \delta^{(3)}(\mathbf{Q}), \\
& A^{(2)}(\mathbf{Q})=\frac{a^{2}}{Q^{2}},
\end{aligned}
$$

while terms $A^{(m)}(\mathbf{Q})$ starting with $m=3$ have the form

$$
\begin{aligned}
A^{(m)}(\mathbf{Q}) & =\frac{(-4 \pi a)^{m}}{(4 \pi)^{2}} \int \frac{d^{3} l_{1}}{(2 \pi)^{3}} \cdots \frac{d^{3} l_{m-2}}{(2 \pi)^{3}} \frac{1}{\mathbf{l}_{1}^{2}} \\
\times & {\left[\frac{1}{\left(\mathbf{l}_{1}-\mathbf{l}_{2}\right)^{2}} \frac{1}{\left(\mathbf{l}_{2}-\mathbf{l}_{3}\right)^{2}} \cdots \frac{1}{\left(\mathbf{l}_{m-2}-\mathbf{Q}\right)^{2}}\right] . }
\end{aligned}
$$

Here, $\mathbf{Q}=\mathbf{p}^{\prime}-\mathbf{p}$ denotes the three-momentum transfer between the incoming and outgoing nucleons and $Q=|\mathbf{Q}|$. The third term, for example, reads

$$
A^{(3)}(\mathbf{Q})=-\frac{\pi}{2} \frac{a^{3}}{Q} .
$$

Upon Fourier transforming we find

$$
A^{(2)}(r)=\frac{a^{2}}{4 \pi r}, \quad A^{(3)}(r)=-\frac{a^{3}}{4 \pi r^{2}} .
$$

These are the first two cases of the well-known form for the $m$ th term

$$
A^{(m)}(r)=-\frac{a}{4 \pi}\left(-\frac{a}{r}\right)^{m-1}
$$

However, a problem occurs when trying to derive the forth term from the momentum-space integral 8

$$
A^{(4)}(\mathbf{Q})=\int \frac{d^{3} l_{1}}{(2 \pi)^{3}} \frac{d^{3} l_{2}}{(2 \pi)^{3}} \frac{(4 \pi)^{2} a^{4}}{\mathbf{l}_{1}^{2}\left(\mathbf{l}_{1}-\mathbf{l}_{2}\right)^{2}\left(\mathbf{l}_{2}-\mathbf{Q}\right)^{2}} .
$$

Since the integral itself is dimensionless, and yet depends on only one dimensionful parameter, $Q$, it should come as no surprise that the result of the integral is independent of that dimensionful parameter. Indeed, one finds, after introducing the variable $z=l_{2} / Q$,

$$
A^{(4)}(\mathbf{Q})=a^{4}\left(1+\int_{1}^{\infty} \frac{d z}{z}\right) .
$$

The Fourier transform of the first, constant, term is a deltafunction in configuration space, which does not match the expected $r$-space expression $a^{4} / 4 \pi r^{3}$. Moreover, the remaining

\footnotetext{
${ }^{4}$ We do not consider the isoscalar $\pi N$ scattering length, since it is unnaturally small both due to its chiral suppression and due to significant numerical cancellations.
}

integral in Eq. (13) is divergent. The easiest way to regularize it is to introduce a finite upper limit in the integration, $z_{\max }=\mu / Q$, which, equivalently, translates to a finite cutoff in $l_{2}$. In this way, through the regularization of the integral, the momentum transfer $Q$ again appears in the expression.

The necessity for regularization of the integral implies, from an EFT perspective, the need to introduce a $\pi d$ counterterm in order to parameterize the short-distance physics which is affecting the result. Its operator structure is a constant in momentum space. This introduces a free parameter: the strength of the finite piece of the counterterm. To simplify the notation we may absorb the constant in the expression given above into this free parameter. We then find

$$
A^{(4)}(\mathbf{Q})=-a^{4} \log \frac{Q}{\mu}+\frac{f_{0}(\mu)}{32 \pi^{2}},
$$

where $f_{0}$ is a $\mu$-dependent parameter. Note that the factor $32 \pi^{2}$ appears here due to our conventions for the definition of the individual terms in the MSS according to Eqs. (1) and (8).

The first term in Eq. (14), relative to $A^{(2)}$, is then of order

$$
a^{2} Q^{2} \sim \frac{M_{\pi}^{4}}{64 \pi^{2} F_{\pi}^{4}}=4 \pi^{2}\left(\frac{M_{\pi}}{4 \pi F_{\pi}}\right)^{4}
$$

where we have counted $Q \sim M_{\pi}$. The suppression of $A^{(4)}$ relative to $A^{(2)}$ is therefore $4 \pi^{2}$ less than the naive-dimensionalanalysis (NDA) estimate for such a two-loop effect, a manifestation of the $\pi^{2}$ enhancement alluded to above.

The size of $f_{0}$ is determined by the coefficient of the logarithm in Eq. (14). We thus have

$$
f_{0} \sim 32 \pi^{2} a^{4}=\frac{M_{\pi}^{4}}{128 \pi^{2} F_{\pi}^{8}}=\frac{2 \pi^{2}}{F_{\pi}^{4}}\left(\frac{M_{\pi}}{4 \pi F_{\pi}}\right)^{4} .
$$

$f_{0}$ then, like the other term in Eq. (14), is $2 \pi^{2}$ larger than $\chi \mathrm{PT}$ power counting would have suggested for a counterterm that renormalizes a two-loop diagram. The enhanced (with respect to their $\chi \mathrm{PT}$ estimate) size of the MSS terms therefore potentially drives the existence of larger-than-expected shortdistance effects.

As an example of what happens with higher terms in the MSS, we briefly mention the corresponding result for $A^{(5)}$. Straightforward evaluation gives

$$
A^{(5)}(\mathbf{Q})=a^{5} \frac{\pi}{4}\left(Q-2 \int d l\right) .
$$

The first term in the brackets, which is finite, can be mapped onto the $1 / r^{4}$ term of the MSS via a properly regularized Fourier transform, while the second (divergent) term generates, in principle, another free parameter. Note, however, that this free parameter, linear in $\mu$, can be absorbed into $f_{0}$. This pattern continues, with all terms of even order in the MSS apparently requiring new operator structures to make them finite, while the odd-order terms have finite pieces which map properly onto their coordinate-space expressions, and whose divergent parts can be absorbed into the counterterms generated at the preceding order in the MSS.

There is thus an apparent problem, since the results obtained above would imply that the MSS comes with an infinite 
set of free parameters, and cannot be regarded as predictive. Moreover, these counterterms are larger than one would expect based on $\chi \mathrm{PT}$ counting for the $\pi d$ problem, because the MSS terms themselves are larger than implied by naive application of $\chi$ PT.

\section{Resumming the MSS}

In this section we show how these problems can be resolved, the central finding being that the UV divergences of the MSS cancel exactly once the series is resummed-for all values of $Q$. A direct consequence of this result is that no enhanced counterterms are required in the MSS. The limitations on the validity of this result will be addressed in more detail in the next section.

In order to proceed we first regularize each term in the MSS and then sum the entire series. This allows us to recover the standard result for each term in the series once the regulator is removed and the resummed result re-expanded in powers of $a$.

Our starting point is Eq. (8). Using the fact that

$$
\frac{1}{p^{2}}=\frac{1}{4 \pi} \int d^{3} r \frac{\exp (i \mathbf{p} \cdot \mathbf{r})}{r}
$$

and applying this to each individual propagator in Eq. (8), after integration over all three momenta and angles one obtains, for the $m$ th term in the MSS $(m \geq 2)$

$$
A^{(m)}(\mathbf{Q})=(-a)^{m} \int \frac{d r}{r^{m-3}} \frac{\sin Q r}{Q r} .
$$

This expression exhibits the following types of divergences: first, for $Q=0$ and $m=4$ it becomes singular in the infrared (IR). In practice, this singularity gets tamed automatically once the convolution with the nuclear wave functions is included (cf. Eq. (3)). Second, starting from $m=4$, this expression is singular in the ultraviolet (UV), $r \rightarrow 0$, as mentioned above. We will show in the following that all UV divergences cancel once the MSS is resummed. Finally, this resummation produces a new type of singularity that shows up for $a<0$, and will be discussed in Sect. 5. In what follows we choose a regularization technique which makes the divergences manifest, so that the argument can be presented in a straightforward and clean way.

In particular, we now regularize (19) by IR and UV cutoffs $R$ and $r_{0}$, respectively. Beginning with the evaluation of the Fourier transform at $Q=0$ we see that the regularized version of $A^{(m)}(\mathbf{0})$ is

$$
A^{(m)}(\mathbf{0})=(-a)^{m} \int_{r_{0}}^{R} \frac{d r}{r^{m-3}},
$$

which gives, for $m=4$,

$$
A^{(4)}(\mathbf{0})=a^{4} \log \frac{R}{r_{0}} .
$$

Thus this integral shows a logarithmic divergence as $r_{0} \rightarrow 0$, in full correspondence to what was stated above (cf. the $\log \mu$ in Eq. (14)). On the other hand, for $m>4$ we obtain

$A^{(m)}(\mathbf{0})=(-a)^{m} \int_{r_{0}}^{R} \frac{d r}{r^{m-3}}=\frac{(-a)^{m}}{m-4}\left(\frac{1}{r_{0}^{m-4}}-\frac{1}{R^{m-4}}\right)$.

The sum of all orders beyond order 4 in $A^{(m)}(\mathbf{0})$ is then

$$
\sum_{m>4} A^{(m)}(\mathbf{0})=a^{4}\left\{\log \frac{R+a}{r_{0}+a}+\log \frac{r_{0}}{R}\right\} .
$$

The last, singular, term in this expression cancels exactly with $A^{(4)}(\mathbf{0})$. Thus, we find that the final result at $Q=0$ is $\mathrm{UV}$ finite provided that regularization is carried out and the full series then resummed. After resummation the limit $r_{0} \rightarrow 0$ is finite so that the regulator can be formally removed, if $a>0$. For $a<0$ this is not possible, since one would hit the branch cut of the logarithm in the first term of Eq. (23). However, as long as the scattering length is natural, $|a| \lesssim 1 / \Lambda$, the regulator $r_{0}$ can at least be pushed outside the regime of validity of the theory. In any case there is no need for the inclusion of enhanced (compared to their $\chi \mathrm{PT}$ estimate) counterterms to remove the UV divergences in the resummed expression. The physical implications of this procedure will be discussed in Sect. 5]

The divergence structure of the case $Q \neq 0$ can be reduced to that discussed in the previous paragraph. Let

$$
A^{(m)}(\mathbf{Q})=A^{(m)}(\mathbf{0})+\delta A^{(m)}(\mathbf{Q}) .
$$

The terms $A^{(m)}(\mathbf{0})$ were already dealt with above. In addition, $\delta A^{(m)}(\mathbf{Q})$ is UV finite for $m=4$ and $m=5$, however, starting from $m=6$ also these terms diverge. Let

$$
\begin{aligned}
\delta \hat{A}(\mathbf{Q}) & =\sum_{m>5} \delta A^{(m)}(\mathbf{Q}) \\
& =\sum_{m>5}(-a)^{m} \int_{r_{0}}^{R} \frac{d r}{r^{m-3}}\left(\frac{\sin Q r}{Q r}-1\right) .
\end{aligned}
$$

In order to proceed we now expand $\sin Q r$ in a power series around $Q=0$ and study each term individually. We get

$$
\delta \hat{A}(\mathbf{Q})=\sum_{n=1}^{\infty} \sum_{m>5}(-a)^{m} \frac{(-1)^{n} Q^{2 n}}{(2 n+1) !} \int_{r_{0}}^{R} d r r^{3-m+2 n} .
$$

This expression becomes UV singular for $m \geq 2 n+4$. For those terms we may write

$$
\begin{aligned}
\delta \hat{A}(\mathbf{Q})_{\operatorname{sing}} & =\sum_{n=1}^{\infty} \sum_{l=1}^{\infty}(-a)^{l+2 n+3} \frac{(-1)^{n} Q^{2 n}}{(2 n+1) !} \int_{r_{0}}^{R} \frac{d r}{r^{l}} \\
& =\left(\frac{\sin Q a}{Q a}-1\right) \sum_{l=1}^{\infty}(-a)^{l+3} \int_{r_{0}}^{R} \frac{d r}{r^{l}} .
\end{aligned}
$$

The last sum is the same one we encountered in summing the terms $A^{(m)}(0)$ (see Eq. (20) ) from $m=4$ to $\infty$. We may therefore follow the same steps applied in that case to obtain

$$
\delta \hat{A}(\mathbf{Q})_{\operatorname{sing}}=\left(\frac{\sin Q a}{Q a}-1\right) a^{4} \log \frac{R+a}{r_{0}+a},
$$


which again has a smooth limit for $r_{0} \rightarrow 0$-as long as $a>0$. Thus we have demonstrated that, for all values of $Q$, the UV divergences of the MSS cancel exactly once the series is resummed. Thus, apparently no counterterms are required and the MSS is predictive. No additional information on shortdistance physics is needed in order for it to render a sensible prediction, as long as we regularize it, and then resum. The UV regulator can then be removed.

Thus far we have been able to sum the singular terms and construct an explicit momentum-space expression for them after summation. This is not, however, the full sum of the MSS, which also includes those terms whose Fourier transform is well-defined without any need to introduce a regulator. In order to derive the full result we solve the integral equation (2) and hence obtain an expression for the full sum of the MSS in coordinate and momentum space.

We can, under quite general conditions, solve Eq. (2) by a function

$$
2 T\left(\mathbf{p}^{\prime}, \mathbf{p}\right)=2(4 \pi)^{2} A(\mathbf{Q})
$$

The function $A$ is most easily computed by taking the inverse Fourier transform of Eq. (2) and applying the convolution theorem. This produces [15]

$$
A(r)=-\frac{a}{4 \pi}-\frac{a}{r} A(r),
$$

such that, as was shown long ago [2,3],

$$
A(r)=-\frac{a r}{4 \pi(r+a)}=-\frac{a}{4 \pi}+\frac{a^{2}}{4 \pi r} \sum_{n=0}^{\infty}\left(-\frac{a}{r}\right)^{n},
$$

and thus each term in the MSS contributes one order in a geometric series in $a / r$.

Clearly, the final expression given in Eq. (31) is a very efficient and useful representation of the MSS. It is not singular as $r \rightarrow 0-$ at least as long as $a>0$ - although the individual terms of the sum are increasingly singular. The evaluation of the expectation value of the full sum with nuclear wave functions is straightforward-again, as long as $a>0$.

The easiest way to obtain the resummed MSS in momentum space is to perform a Fourier transform of the resummed $r$-space expression 31. This yields

$$
A(\mathbf{Q})=-\frac{(2 \pi)^{3}}{4 \pi} a \delta^{(3)}(\mathbf{Q})+\frac{a^{2}}{Q^{2}}-\frac{a^{3}}{Q} f(a Q),
$$

with

$$
\begin{aligned}
f(y) & =\int_{0}^{\infty} d x \frac{\sin x}{x+y} \\
& =\operatorname{Ci}(y) \sin y+\frac{1}{2} \cos y(\pi-2 \operatorname{Si}(y)),
\end{aligned}
$$

where we used the following definitions of the sine and cosine integral functions

$$
\operatorname{Ci}(y)=-\int_{y}^{\infty} d t \frac{\cos t}{t}, \quad \operatorname{Si}(y)=\int_{0}^{y} d t \frac{\sin t}{t} .
$$

In this context it is important to note that the function $\mathrm{Ci}(y)$, appearing in Eq. (33), has a branch point at $y=0$ - it acquires an unphysical cut from $a Q=0$ to $a Q=-\infty$ which enters the physical region for negative values of $a$. This cut appears to be the momentum space analog of the unphysical pole at $r=-a$ of Eq. (31), but it should not be a concern for $a>0$.

Moreover, for small positive $y$, we may expand $f(y)$ in powers of $y$, and so obtain

$$
f(a Q)=\frac{\pi}{2}+(\gamma-1+\log a Q) a Q-\frac{\pi(a Q)^{2}}{4}+\cdots,
$$

where $\gamma$ denotes the Euler-Mascheroni constant. Insertion of this expansion into Eq. 32 produces a power series in $a$ in which all non-analytic terms match with what we found in Sect. 3 Note, however, that in the full, finite expression the logarithmic divergence of Eq. (14) becomes effectively regularized at the scale $1 / a$. To better understand the structure and the coefficients of the term $\propto a^{4}$ in Eq. (32), one may calculate the quadruple-scattering term explicitly starting from the expression 19]

$$
\begin{aligned}
A^{(4)}(\mathbf{Q}) & =a^{4} \int_{Q r_{0}}^{\infty} d x \frac{\sin x}{x^{2}} \\
& =-a^{4}\left(\gamma-1+\log Q r_{0}\right) .
\end{aligned}
$$

This explains how the constant term $\gamma-1$ appears in Eq. (35) and demonstrates that the UV regulator $r_{0}$ is effectively replaced by $a$ due to the resummation procedure described above.

The pole/cut in coordinate/momentum space that appears for $a<0$ for the MSS of meson-nucleus scattering emerges since we focused on isoscalar interactions. Under certain conditions, a different isospin structure can make the pole disappear. To illustrate this point, we consider $\pi^{-} d$ scattering with the $\pi^{-} p \rightarrow \pi^{0} n$ and $\pi^{0} n \rightarrow \pi^{0} n$ channels switched off. In the isospin limit, this can be described with isoscalar and isovector $\pi N$ scattering lengths $a^{+}$and $a^{-}$. The (coordinate space) result for the resummed MSS then reads

$$
A(r)=-\frac{a^{+}}{4 \pi}+\frac{\left(a^{+}\right)^{2}-\left(a^{-}\right)^{2}}{4 \pi\left(r^{2}-\left(a^{+}\right)^{2}+\left(a^{-}\right)^{2}\right)}\left(r-a^{+}\right),
$$

which for $a^{-} \rightarrow 0$ reduces to Eq. 31. Provided that $\left|a^{-}\right|>$ $\left|a^{+}\right|$, the pole disappears and the result for the MSS is welldefined everywhere. Similarly, a pole in the full MSS for $\pi^{-} d$ scattering, with the $\pi^{-} p \rightarrow \pi^{0} n$ and $\pi^{0} n \rightarrow \pi^{0} n$ channels included, would only appear if $\pi N$ interactions were not predominantly of isovector nature.

For this reason, the discussion of the case $a<0$ in which a pole appears in $A(r)$ might appear quite academic. However, as we will show in Sect. 6 this kind of pole does appear when the sum of the $\pi N N$ MSS which contributes to the $N N$ potential is computed.

\section{Interpretation}

The previous section suggests that the MSS has a valid expansion if $0<a Q<1$, so that the expression (35) can be employed and the cut in $f(y)$ does not enter the physical $(Q>0)$ region. In coordinate space these conditions correspond to $r>$ $|a|$ and $a>0$. In case of $a<0$, however, a pole (cut) appears in 
the resummed coordinate (momentum) space expression. This issue will be addressed at the end of this section.

In general, the fact that a resummation gives a well-defined answer does not necessarily mean that it gives the correct answer. This was recently stressed in Ref. [23]. In particular, one might be concerned that the sensitivity to short-distance physics in the MSS necessitated by the perturbative treatment with a point-like $\pi N$ interaction indicates that the regularized, resummed result of Sect. 4 obtained in the limit of $r_{0} \rightarrow 0$ is not correct and thus strongly reduces the predictive power of our theoretical approach.

In general, the $\pi N$ interaction has a certain range, and the $t$-matrix is not momentum independent. Here we therefore introduce a regularized $\pi N$ interaction, which is non-pointlike at scale $\Lambda$, and discuss the conditions under which the results of the previous section are accurate. In this way the scale $\Lambda$ is completely equivalent to the regulator $r_{0}$ introduced in the previous section, however, it has a physical interpretation of the scale that limits the validity of the theory. Thus the difference between the results with a finite $\Lambda$ and those in the limit $\Lambda \rightarrow \infty$ provides a measure of the consistency of the scheme. This difference, if the scheme is self-consistent, should be less than the contribution of the leading contact operator. For example, for pion-deuteron scattering investigated within $\chi \mathrm{PT}$ the first contact term contributes at $\mathcal{O}\left(p^{2}\right)$ (or parametrically $\left.m_{\pi}^{2} /\left(f_{\pi}^{4} \Lambda^{2}\right)\right)$ and its size was estimated to be around $5 \%$ relative to the leading double-scattering diagram [10,12].

We now write

$$
t_{\pi N}(\mathbf{p}, \mathbf{0})=-4 \pi a \hat{g}\left(\frac{|\mathbf{p}|}{\Lambda}\right)
$$

with $\hat{g}(x) \rightarrow 1$ as $x \rightarrow 0$. The amplitude for scattering of the zero-momentum pion from the $N N$ system still takes the form 29, but now

$$
A(r)=-\frac{a}{4 \pi}-\frac{a}{r} g(r) A(r)
$$

with

$$
g(r)=4 \pi r \int \frac{d^{3} k}{(2 \pi)^{3}} e^{-i \mathbf{k} \cdot \mathbf{r}} \frac{1}{\mathbf{k}^{2}} \hat{g}^{2}\left(\frac{|\mathbf{k}|}{\Lambda}\right) .
$$

Thus, in the presence of the regulator $g$,

$$
A(r)=-\frac{a r}{4 \pi(r+a g(r))} .
$$

If, for illustrative purposes, we take

$$
\hat{g}(x)=\frac{1}{\sqrt{1+x^{2}}}
$$

then

$$
g(r)=1-e^{-\Lambda r},
$$

and so $g(r) \rightarrow 1$ as $r \rightarrow \infty$, but $g(r) \rightarrow \Lambda r$ as $r \rightarrow 0$. While the details of this result are specific to the form 42), the disappearance of regulator effects in the infrared and the appearance of additional powers of $r$ in the ultraviolet is a general feature.

If $A(r)$ is evaluated perturbatively based on the expansion of Eq. 411, we have (cf. Eq. 31)

$$
A^{(m)}(r)=-\frac{a}{4 \pi}\left(-\frac{a g(r)}{r}\right)^{m-1}
$$

with $m \geq 1$. Observe that introducing the form factor $g$ with the cutoff $\Lambda$ leads to results completely equivalent to those obtained using a sharp cutoff that were discussed in detail in the previous section. In particular, the individual terms in the MSS again appear to be enhanced relative to their $\chi \mathrm{PT}$ estimates: for example, the momentum-space form of the quadruple-scattering term exhibits the behavior $A^{(4)}(\mathbf{Q}) \sim-a^{4} \log Q / \Lambda$ discussed in Sect. 3 And, once again, the resummed result (41) is much less $\Lambda$-dependent than the individual terms in the MSS. The limit $\Lambda \rightarrow \infty$ exists there due to the cancellations of the UVdivergent terms derived in the previous section. But, the form (44) makes explicit that it is the behavior $g(r) \rightarrow \Lambda r$ as $r \rightarrow 0$ which guarantees that none of the terms in the MSS diverge as $r \rightarrow 0$. It is crucial to observe that this regularization only prevails if $\Lambda$ is kept finite, as an effective scale representing the range of validity of the theory, when Eq. (41) is expanded in powers of $a$ and Fourier transformed to momentum space. Although, of course, if one is interested in the long-distance $(r>|a|)$ form of each contribution to the MSS in $r$-space, the limit $\Lambda \rightarrow \infty$ can be safely taken in each of the terms in Eq. (44).

In physical terms we anticipate working in perturbative meson-nucleon systems where $|a| \sim 1 / \Lambda$, and so we can resum the series in $a / r$ to infinite order with $\Lambda$ being large but finite. This yields a well-defined mathematical procedure. In the resummed expression (see Eq. (41)) the limit $\Lambda \rightarrow \infty$ exists. However, the question is whether it can be safely taken. In other words, how does the physics of the finite $\Lambda$ affect the EFT result for the MSS? The difference between the $\Lambda \rightarrow \infty$ and finite- $\Lambda$ results for $A(r)$ is

$$
\Delta A \equiv A(r)-A_{\Lambda \rightarrow \infty}(r)=-\frac{a^{2} r(1-g(r))}{4 \pi(r+a)(r+a g(r))} .
$$

For $\pi d$ scattering within $\chi \mathrm{PT}$, the convolution of Eq. 45 with the (pionful) deuteron wave functions obtained in chiral EFT [24] results in an effect of less than $3 \%$ to the piondeuteron scattering length (with $\Lambda \sim M_{\rho} \sim 800 \mathrm{MeV}$ ). This is fully in line with the estimate of the contact operator at $\mathcal{O}\left(p^{2}\right)$. Thus, taking the limit $\Lambda \rightarrow \infty$ is justified in the resummed expression 41], and the results of the previous section are correct. No enhanced counterterms are therefore required in the case of the MSS with a natural scattering length.

We now focus our attention on the pole at $r=-a$, relevant in the case $a<0$. In Eq. (41) the analog of this pole occurs as the solution of

$$
r=-a g(r),
$$

which can be found in an analytic form for the form factor chosen in Eq. (43). In this case, Eq. (46) can be rewritten in the form

$$
\Lambda(r-|a|) e^{\Lambda(r-|a|)}=-\Lambda|a| e^{-\Lambda|a|},
$$

whose solution is

$$
r=\frac{1}{\Lambda}\left(\Lambda|a|+W\left(-\Lambda|a| e^{-\Lambda|a|}\right)\right),
$$

where $W(z)$ is the Lambert $W$-function defined via $z=W e^{W}$ for any complex number $z$. It has a branch-cut discontinuity in the complex plane $z$ running from $-\infty$ to $-1 / e$. In particular, the branch point $z=-1 / e$ corresponds to $\Lambda|a|=$ 


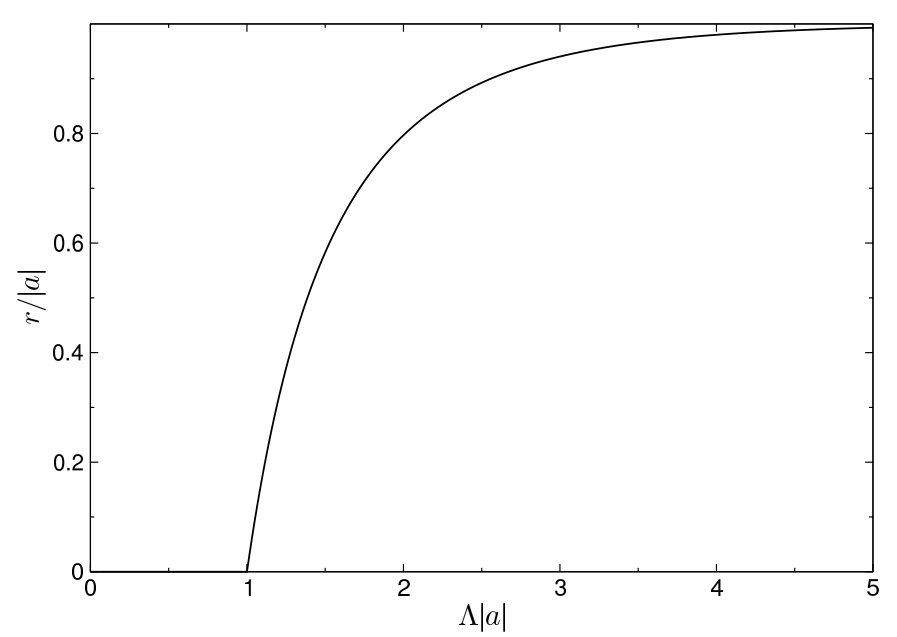

Fig. 2. Position of the pole at $r=-a g(r)$ for $a<0$. The pole is at $r=0$ if $\Lambda|a| \leq 1$.

1 in Eq. (48). As a consequence, for $\Lambda|a| \leq 1$ we have $W\left(-\Lambda|a| e^{-\Lambda|a|}\right)=-\Lambda|a|$ and the pole is at the origin. In contrast, for $\Lambda|a|>1$ the pole moves away from 0 and is located within the interval $r \in(0,|a|]$, the upper limit being reached in the situation $\Lambda|a| \gg 1$. The corresponding trajectory of the pole as a function of $\Lambda|a|$ is depicted in Fig. 2.

For a natural value of the meson-nucleon scattering length the pole only arises due to short-distance $(r \sim 1 / \Lambda)$ parts of the MSS diagrams, and these parts of the diagrams cannot be regarded as a reliable prediction of the EFT. Or, phrased differently, we can assume that for values of $r$ in the applicability range of the theory the resummed MSS delivers reliable results as long as $a$ is natural.

An unnaturally large scattering length $a$, i.e. $|a| \gg 1 / \Lambda$, indicates the presence of a shallow meson-nucleon bound or virtual state. In this case, once $r \sim a$ it is not appropriate to write $t=-4 \pi a$, even if $r \gg 1 / \Lambda$. Instead we must at least resum unitarity corrections to the meson-nucleon amplitude, which should remove the pole in the case of $a<0$ or shift it towards the origin. It remains to be seen in each particular case whether it suffices to keep only the leading unitarity term $(\propto i k)$ in the amplitude, or if a non-perturbative treatment of finite-range corrections is needed as well.

As a possible example of this situation one may consider $K d$ scattering where at least one of the $K N$ scattering lengths is of the order of $1 \mathrm{fm}$ and negative, see, e.g., Ref. [16]. In this case it appears useful to employ a non-relativistic effective field theory [16,17] which operates with the threshold parameters ( $K N$ scattering lengths) and reproduces the result of the $K d$ MSS [15]. The usefulness of the theory is based on the separation of two distinct scales. While the $N N$ interaction is mediated by one-pion exchange at large distances, the $K N$ interaction is governed by the two-pion exchange, which justifies the treatment of $K N$ interactions as point-like. The range of validity of the approach is thus $\Lambda \sim 300 \mathrm{MeV}$. In spite of the large scattering length, for such a small $\Lambda$ the product $\Lambda|a|$ is still close to 1 so that the pole might still be near the origin 5 .

\footnotetext{
${ }^{5}$ Note that in the real world the $K N$ scattering lengths are strongly absorptive so that the pole cannot be on the real axis.
}

Therefore we do not expect the counterterm to be enhanced in this case either. Meanwhile, keeping the unitarity corrections at distances $r \sim a$ appears necessary.

\section{Remarks on the multiple-scattering series in the nucleon-nucleon potential}

We now turn our attention to the MSS in the nucleon-nucleon potential. After a pion is emitted from one nucleon it can propagate in the $N N$ system via the full MSS, before being reabsorbed on the other nucleon. Therefore, also in the nucleonnucleon potential, diagrams enhanced compared to their $\chi \mathrm{PT}$ order in a similar manner to that discussed above appear. In this section we discuss the consequences of this aspect of the MSS for chiral EFT computations of the $N N$ potential. Here, we leave aside the issues associated with non-perturbative renormalization of that potential. The interested reader may consult Refs. [23, 25, 26, 27, 28, 29] and references therein for a sample of different views on this issue. In this work we are interested in two particular questions regarding the meson-exchange diagrams which generate the long-distance (van-der-Waals in the chiral limit) part of the potential: are the MSS diagrams enhanced? If so, what does that imply for the scale at which a perturbative expansion of the long-distance potential breaks down?

A key difference between the multiple-scattering terms in $\pi$-nucleus scattering and the nucleon-nucleon potential is that the meson propagator $1 /\left(\mathbf{l}_{i}-\mathbf{l}_{j}\right)^{2}$ as it appears, e.g., in Eq. (12) is to be replaced by $1 /\left(\left(\mathbf{l}_{i}-\mathbf{l}_{j}\right)^{2}+M_{\pi}^{2}\right)$. The expression for the corresponding one-loop term is then proportional to [30, 31]

$$
\frac{1}{2 Q} \arctan \frac{Q}{2 \mu}
$$

with $\mu=M_{\pi}$. In the kinematics for pion-nucleus scattering we need to choose $\mu=0$, since here the energy transfer and the meson mass cancel exactly in the pion propagator. Once this limit is taken an additional factor of $\pi / 2$ appears, and Eq. (49) reduces to Eq. (9). Thus, the enhancement of this graph in the $N N$ scattering potential is not the $\pi^{2}$ we found for $\pi$-nucleus scattering, but we do still have enhancement by a factor of $\pi$ over the NDA estimate of this graph.

This enhancement is phenomenologically important. It is well known that the strongest contribution to the two-pionexchange potential up to $\mathrm{N}^{2} \mathrm{LO}$ emerges from the subleading triangle diagram. While nominally subleading, the corresponding central isoscalar potential appears to be an order of magnitude stronger than all the other two-pion-exchange contributions. This unnaturally large contribution can be traced back to the aforementioned triangle graphs' enhancement by one power of $\pi$, together with the numerically large value of the low-energy constant (LEC) $c_{3}$, which parameterizes the subleading $\pi \pi N N$ vertex and is largely saturated by the $\Delta$ isobar [32]. These observations provide a strong motivation to take a closer look at higher-order terms in the MSS beyond the triangle diagram. Although one expects that potentials generated by the exchange of a large number of pions are exponentially suppressed at distances $r \sim M_{\pi}^{-1}$, one should keep in mind their singular, van-der-Waals-like behavior at shorter distances. 

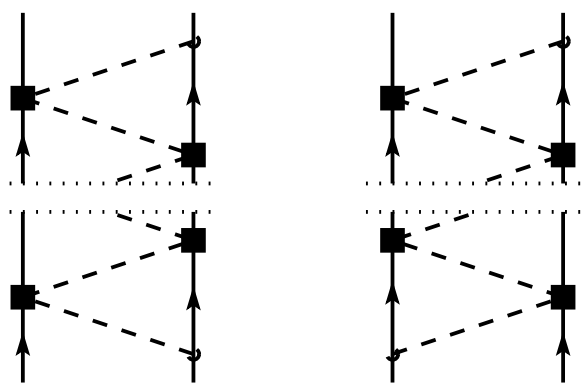

Fig. 3. Time-ordered MSS-diagram contribution to the nucleonnucleon potential. Solid dots (filled rectangles) refer to the leadingorder vertices from the effective Lagrangian proportional to $g_{A}\left(c_{i}\right)$.

We will see below how these competing features influence the convergence of the chiral expansion for this particular set of diagrams.

To be specific, we consider time-ordered two-nucleon diagrams in the MSS as shown in Fig. 3. In this exploratory study, we restrict ourselves to the isoscalar part of the subleading $\pi \pi N N$ vertex

$$
\left\langle\mathbf{l}_{1}, i|H| \mathbf{l}_{2}, j\right\rangle=\frac{\delta_{i j}}{F_{\pi}^{2} \sqrt{\omega_{1} \omega_{2}}}\left(2 c_{1} M_{\pi}^{2}+c_{3} \mathbf{l}_{1} \cdot \mathbf{l}_{2}\right),
$$

where $\mathbf{l}_{1,2}$ denote the pion momenta, $i$ and $j$ are the pion isospin quantum numbers and $\omega_{1,2} \equiv \sqrt{\mathbf{l}_{1,2}^{2}+M_{\pi}^{2}}$ are the free pion energies. The potential corresponding to the left diagram in Fig. 3, where an even number of pion exchanges occurs, is given by

$$
\begin{aligned}
V^{n \pi}(\mathbf{q}) & =-\frac{3 g_{A}^{2}}{8 F_{\pi}^{2 n}} \int \frac{d^{3} l_{1}}{(2 \pi)^{3}} \ldots \frac{d^{3} l_{n}}{(2 \pi)^{3}}(2 \pi)^{3} \\
& \times \delta^{(3)}\left(\mathbf{l}_{1}+\mathbf{l}_{2}+\ldots+\mathbf{l}_{n}-\mathbf{q}\right) \frac{\boldsymbol{\sigma}_{2} \cdot \mathbf{l}_{n} \boldsymbol{\sigma}_{2} \cdot \mathbf{l}_{1}}{\omega_{1}^{2} \omega_{2}^{2} \ldots \omega_{n}^{2}} \\
& \times\left(2 c_{1} M_{\pi}^{2}-c_{3} \mathbf{l}_{1} \cdot \mathbf{l}_{2}\right)\left(2 c_{1} M_{\pi}^{2}-c_{3} \mathbf{l}_{2} \cdot \mathbf{l}_{3}\right) \\
& \times \ldots\left(2 c_{1} M_{\pi}^{2}-c_{3} \mathbf{l}_{n-1} \cdot \mathbf{l}_{n}\right)+1 \leftrightarrow 2 .
\end{aligned}
$$

Clearly, the integrals entering this expression are UV divergent. The divergences, however, are absorbed into $4 N$ contact operators, and so do not affect $r$-space expressions for the finiterange part of the potential that we are discussing here. The $r$ space representation of the potential can be obtained straightforwardly, leading to

$$
\begin{aligned}
V^{n \pi}(\mathbf{r}) & =\frac{3 g_{A}^{2}}{4 F_{\pi}^{2 n}} \nabla_{1} \cdot \nabla_{n}\left(2 c_{1} M_{\pi}^{2}+c_{3} \nabla_{1} \cdot \nabla_{2}\right) \\
& \times\left(2 c_{1} M_{\pi}^{2}+c_{3} \nabla_{2} \cdot \nabla_{3}\right) \ldots \\
& \times\left(2 c_{1} M_{\pi}^{2}+c_{3} \nabla_{n-1} \cdot \nabla_{n}\right) \\
& \times\left. U\left(r_{1}\right) U\left(r_{2}\right) \ldots U\left(r_{n}\right)\right|_{r_{1}=r_{2}=\ldots=r_{n}=r}
\end{aligned}
$$

with

$$
U(r)=\frac{1}{4 \pi r} e^{-M_{\pi} r}
$$

being the usual Yukawa function. After evaluating the derivatives, one ends up with the isoscalar central potential

$$
\begin{aligned}
V^{n \pi}(\mathbf{r}) & =\frac{3 g_{A}^{2}}{4\left(4 \pi F_{\pi}^{2}\right)^{n}} \frac{e^{-n x}}{r^{3 n}}\left[\sum_{m=0}^{n-2} \sum_{l=0}^{m} y_{m l}^{n}\left(2 c_{1} x^{2}\right)^{n-m-1}\right. \\
& \times c_{3}^{m}(1+x)^{2(m+1-l)}\left(2+2 x+x^{2}\right)^{l} \\
& \left.+c_{3}^{n-1}\left(\left(2+2 x+x^{2}\right)^{n}+2(1+x)^{n}\right)\right]
\end{aligned}
$$

Here we introduced a dimensionless variable $x \equiv M_{\pi} r$ and combinatorial coefficients $y_{m l}^{n}$ whose explicit values can be derived straightforwardly. In a completely similar way, one finds that the second diagram in Fig. 3, where an odd number of pion exchanges takes place, gives rise to the isovector tensor and spin-spin potential

$$
\begin{aligned}
V^{n \pi}(\mathbf{r}) & =\frac{g_{A}^{2}}{4\left(4 \pi F_{\pi}^{2}\right)^{n}} \boldsymbol{\tau}_{1} \cdot \boldsymbol{\tau}_{2} \frac{e^{-n x}}{r^{3 n}}\left(\boldsymbol{\sigma}_{1} \cdot \hat{\mathbf{r}} \boldsymbol{\sigma}_{2} \cdot \hat{\mathbf{r}}\right. \\
& \times\left[\sum_{m=0}^{n-2} \sum_{l=0}^{m} y_{m l}^{n}\left(2 c_{1} x^{2}\right)^{n-m-1} c_{3}^{m}(1+x)^{2(m+1-l)}\right. \\
& \times\left(2+2 x+x^{2}\right)^{l}+c_{3}^{n-1}\left(\left(2+2 x+x^{2}\right)^{n}\right. \\
& \left.\left.\left.+(1+x)^{n}\right)\right]-\boldsymbol{\sigma}_{1} \cdot \boldsymbol{\sigma}_{2} c_{3}^{n-1}(1+x)^{n}\right)
\end{aligned}
$$

where $n=2 k+1, k \in \mathbb{N}$.

As expected, based on the discussion at the start of this section, each extra loop in the MSS generates a power of $1 /\left(4 \pi F_{\pi}^{2}\right)$, rather than the $1 /\left(4 \pi F_{\pi}\right)^{2}$ that is usually assumed in $\chi \mathrm{PT}$. This is the way the "enhancement" of MSS diagrams plays out in the $N N$ potential. These contributions to the potential, $V^{n \pi}(\mathbf{r})$, take a particularly simple form if either $c_{1}$ or $c_{3}$ is set to zero. In particular, the central isoscalar potential in Eq. (54) reads in these two limits

$$
\begin{aligned}
& V_{c_{1}}^{n \pi}(\mathbf{r})=\frac{3 g_{A}^{2}}{8\left(2 \pi F_{\pi}^{2}\right)^{n}} \frac{e^{-n x}}{r^{3 n}}\left(c_{1} x^{2}\right)^{n-1}(1+x)^{2} \\
& V_{c_{3}}^{n \pi}(\mathbf{r})=\frac{3 g_{A}^{2} c_{3}^{n-1}}{4\left(4 \pi F_{\pi}^{2}\right)^{n}} \frac{e^{-n x}}{r^{3 n}}\left[\left(2+2 x+x^{2}\right)^{n}+2(1+x)^{n}\right] .
\end{aligned}
$$

Resumming the resulting geometric series leads to the following closed-form expressions for the potentials

$$
\begin{aligned}
V_{c_{1}}(\mathbf{r}) & =\frac{3 g_{A}^{2} c_{1} M_{\pi}^{2}}{32 \pi^{2} F_{\pi}^{4}} \frac{e^{-2 x}}{r^{4}}(1+x)^{2} \frac{1}{1-\frac{c_{1}^{2} M_{\pi}^{4}}{4 \pi^{2} F_{\pi}^{4}} \frac{e^{-2 x}}{r^{2}}}, \\
V_{c_{3}}(\mathbf{r}) & =\frac{3 g_{A}^{2} c_{3}}{64 \pi^{2} F_{\pi}^{4}} \frac{e^{-2 x}}{r^{6}}\left[\frac{2(1+x)^{2}}{1-\frac{c_{3}^{2}}{16 \pi^{2} F_{\pi}^{4}} \frac{e^{-2 x}}{r^{6}}(1+x)^{2}}\right. \\
& \left.+\frac{\left(2+2 x+x^{2}\right)^{2}}{1-\frac{c_{3}^{2}}{16 \pi^{2} F_{\pi}^{4}} \frac{e^{-2 x}}{r^{6}}\left(2+2 x+x^{2}\right)^{2}}\right] .
\end{aligned}
$$

Both $V_{c_{3}}$ and $V_{c_{1}}$ feature poles at finite values of $r>0$ similar to what we observed for the MSS of meson-nucleus scattering 


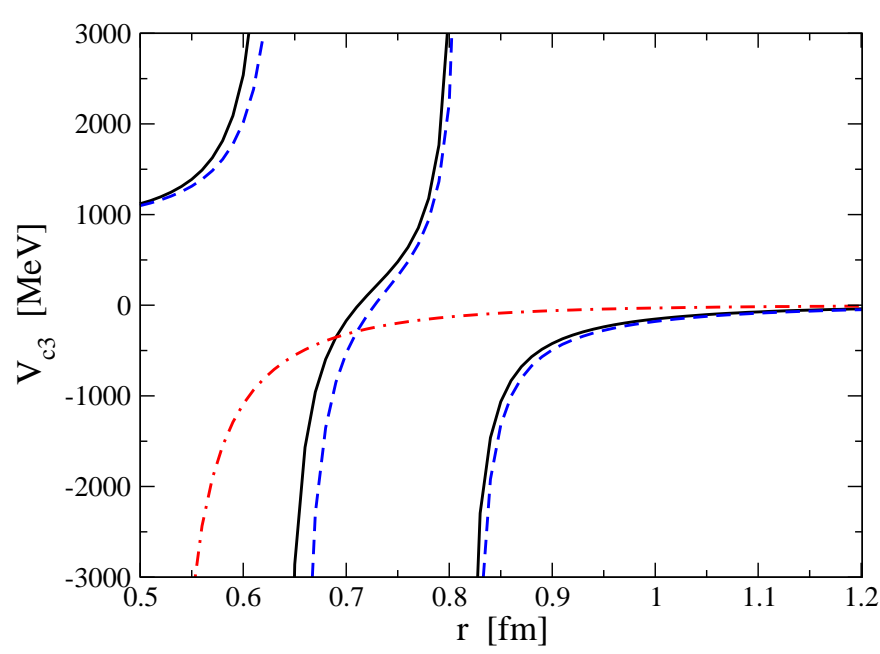

Fig. 4. Resummed potential $V_{c_{3}}$ from Eq. (57). The solid (dashed) line shows the potential for $c_{3}=-3.87 \mathrm{GeV}^{-1}$ and $M_{\pi}=138$ $\mathrm{MeV}\left(M_{\pi}=138 / 4 \mathrm{MeV}\right)$, while the dashed-dotted line depicts the potential for $c_{3}=-1 \mathrm{GeV}^{-1}$ and $M_{\pi}=138 \mathrm{MeV}$.

for $a<0-$ c.f. Eq. 31 - only here the appearance of the poles is independent of the sign of the scattering parameters $c_{i}$. These unphysical poles are non-perturbative phenomena resulting from the partial resummation of the amplitude.

One may view the location of the poles as a measure of the breakdown scale of the chiral expansion for the considered class of diagrams. It is comforting to see that the pole in $V_{c_{1}}$ is located at a rather short distance, namely

$$
r \sim \frac{\left|c_{1}\right| M_{\pi}^{2}}{2 \pi F_{\pi}^{2}} \sim 0.05 \mathrm{fm}
$$

and is shifted to the origin in the chiral limit. On the other hand, the pole positions in $V_{c_{3}}$ are not protected by powers of $M_{\pi}$ and can be estimated by

$$
r \sim \mathcal{O}\left(\left(\frac{\left|c_{3}\right|}{\pi F_{\pi}^{2}}\right)^{1 / 3}\right) \sim \mathcal{O}(1 \mathrm{fm})
$$

using the value $c_{3}=-3.87 \mathrm{GeV}^{-1}$ from the $\mathcal{O}\left(Q^{2}\right)$ fit to $\pi N$ threshold coefficients of Ref. [33]. Numerically, the poles appearing in the two terms of $V_{c_{3}}$ in Eq. (57) are found to be located at

$$
r \simeq 0.63 \mathrm{fm}, \quad r \simeq 0.81 \mathrm{fm},
$$

see Fig. 4 As shown in this figure, the behavior of the resummed potential at short distances and the pole positions only weakly depend on the values of the pion mass in the case of $V_{c_{3}}$. It is somewhat surprising that the chiral expansion for the pion-exchange potential breaks down at the relatively large distance of $\sim 0.8 \mathrm{fm}$. This behavior is, to a large extent, caused by the already discussed enhancement of the diagrams in the MSS, where loops generate inverse powers of $4 \pi F_{\pi}^{2}$ rather than $\left(4 \pi F_{\pi}\right)^{2}$, see Eq. (59). An additional enhancement occurs due to the large numerical value of the LEC $c_{3}$. As shown in [33], this LEC takes a much more natural value of the or$\operatorname{der} c_{3} \sim-1 \mathrm{GeV}^{-1}$ once the $\Delta$-isobar is explicitly taken into account. Therefore, one might expect that the unphysical poles

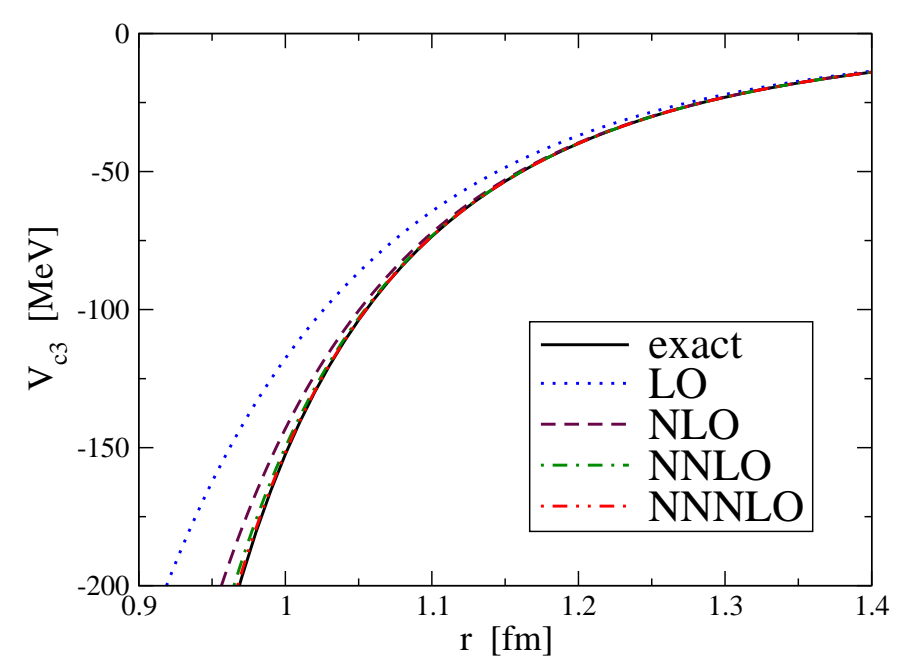

Fig. 5. Convergence of the MSS for $V_{c_{3}}$.

in the potential are shifted closer to the origin in the $\Delta$-full approach, see Fig. 4. For example, setting $c_{3}=-1 \mathrm{GeV}^{-1}$ the poles are shifted to

$$
r \simeq 0.41 \mathrm{fm}, \quad r \simeq 0.52 \mathrm{fm}
$$

The above arguments therefore suggest that the breakdown scale for the chiral expansion of the pion-exchange potential is in the range $r \sim 0.5 \ldots 0.8 \mathrm{fm}$. This estimate agrees well with the findings of various recent studies, see e.g. [34, 35, 36, 37]. In this context it should be stressed, however, that it would be insufficient to include the $\Delta$ as a static field, for then its inclusion would do nothing but to restore the original strength of $c_{3}-$ see discussion in Refs. [38,33]. Thus, only a $\Delta$ with retained recoils could help, which makes sense only if also the nucleons are treated as non-static. The corresponding calculations are very involved and go beyond the scope of this paper.

Given the rather large value of the breakdown scale $r \sim$ $0.8 \mathrm{fm}$, one might worry about the convergence of the chiral expansion for the potential at distances of the order $1 \ldots 2 \mathrm{fm}$. Fortunately, the convergence of the MSS appears to be rather fast, see Fig. 5. In particular, one observes that the potential is already very well described by the subleading term in the MSS. Clearly, the reason for this fast convergence is due to the exponential falloff of the potential at distances large compared to the exchanged mass.

It must be stressed that, while the results obtained here for the resummed potential provide qualitative insights, they are by no means a complete $\chi \mathrm{PT}$ calculation. In addition to the omitted baryon recoils, we only picked out time-ordered graphs that give rise to the MSS. In contrast to near-threshold 6 pion-nucleus scattering, where time-ordered graphs involving two or more pions in the intermediate states can be represented by contact operators [39], there is, strictly speaking, no justification for neglecting such diagrams in the $N N$ potential. On the other hand, the neglected time-ordered graphs in most cases are suppressed by powers of $\pi$ compared to the one in

\footnotetext{
${ }^{6}$ More precisely, the argument refers to the case when the momenta of external pions and nucleons are much smaller than $M_{\pi}$.
} 
the MSS. This is because the maximal enhancement by powers of $\pi$ requires that the individual pion exchanges factorize out leading to integrands of the kind $1 /\left(\omega_{1}^{2} \omega_{2}^{2} \ldots \omega_{n}^{2}\right)$. Such a factorization appears in the integrals resulting from the MSS, while the integrands resulting from other topologies normally have a more complicated structure. For example, the leading football two-pion-exchange diagrams involving two WeinbergTomozawa vertices yield $1 /\left(\omega_{1} \omega_{2}\left(\omega_{1}+\omega_{2}\right)\right)$. We can still use the same machinery to obtain the potential in $r$-space employing the integral representation

$$
\frac{1}{\omega_{1} \omega_{2}\left(\omega_{1}+\omega_{2}\right)}=\frac{2}{\pi} \int_{0}^{\infty} d \beta \frac{1}{\left[\omega_{1}^{2}+\beta^{2}\right]\left[\omega_{2}^{2}+\beta^{2}\right]},
$$

on the cost of introducing an additional power of $\pi$ in the denominator. But, then this power of $\pi$ means that ultimately this graph is not enhanced compared to its $\chi \mathrm{PT}$ estimate. Similar arguments apply to the leading triangle and box diagrams contributing to the two-pion exchange potential at NLO: neither of them is enhanced by a power of $\pi$. In some cases, however, a sum of several non-MSS-type time-ordered graphs may lead to factorized expressions. The simplest example is given by subleading two-pion exchange from the triangle diagrams $\propto c_{i}$. In addition to the diagram contributing to the MSS $\propto 1 /\left(\omega_{1}^{2} \omega_{2}^{2}\right)$, the remaining two time-ordered graphs also yield

$$
\frac{1}{\omega_{1}^{2} \omega_{2}\left(\omega_{1}+\omega_{2}\right)}+\frac{1}{\omega_{1} \omega_{2}^{2}\left(\omega_{1}+\omega_{2}\right)}=\frac{1}{\omega_{1}^{2} \omega_{2}^{2}} .
$$

This is the reason why the result in Eq. (56) for $n=2$ actually gives half the chiral NNLO potential. It remains to be clarified whether similar patterns emerge at higher orders in the loop expansion, and therefore whether there are other contributions which are enhanced in a similar fashion to those discussed here.

Indeed, at each order in the chiral expansion, there are many other topologies and contributions that have not been considered here. Again, one can argue that for non-MSS diagrams such as e.g. the correlated two-pion exchange, one generally does not expect enhancement by powers of $\pi$, but this is not a proof. Furthermore, within the class of MSS time-ordered diagrams driven by the $c_{i}$ 's we did not take into account the energy dependence of the $c_{i}$-vertex and have not considered the contributions driven by $c_{4}$. Although all of these points will change the quantitative results for the $N N$ potential, none of them should affect the qualitative conclusion. Poles at $r \sim 0.8 \mathrm{fm}$ can still be expected in the full result, thanks to terms which are exponentially suppressed for $r>1 \mathrm{fm}$, but become comparable to the dominant two-pion-exchange parts of the $N N$ potential as the inter-nucleon distance decreases further.

\section{Conclusion}

The multiple-scattering series in its resummed form has been known and used for decades. In this work we have looked at that result from an EFT point of view, examining the divergence structure of the individual terms of the series in detail. This is of particular importance since a theoretical analysis of modern, high-accuracy, meson-nucleon experiments calls for solid, quantitative understanding of the corresponding amplitudes. The enhancement of MSS terms by powers of $\pi$ compared to their expected $\chi \mathrm{PT}$ size might lead to concerns about a related enhancement of the associated counterterms.

We have demonstrated analytically that, although the EFT integrals for the MSS in momentum space are singular starting from the fourth term, under certain circumstances all UV divergences cancel upon resummation, provided that resummation is executed with a finite regulator in place. The error induced upon taking the regulator to infinity in the end is less than the size of the counterterm that absorbs the dominant shortdistance effects in the $\pi N N$ system-as must be the case if the power counting for that counterterm is valid. Therefore at least in the case of a natural $(|a| \sim 1 / \Lambda)$ or a positive scattering length, e.g. in the $\pi d$ case- no enhanced counterterms are necessary. Counterterms of normal $\chi \mathrm{PT}$ size will still complement the MSS diagrams-as will other $\chi \mathrm{PT}$ graphs which are not of the MSS topology. But, the $r$-space MSS expressions derived a long time ago based on the Fourier transform of the integral equations in momentum space are justified from an EFT point of view as the well-defined sum of a particular class of graphs.

Meanwhile, straightforward evaluation of the MSS in the scattering-length approximation for the meson-nucleon $t$ matrix implies the appearance of an unphysical pole in the $r$ space result (cut in the momentum-space result) in the case $a<0$. For a natural scattering length this pole is outside the range of applicability of the theory. However, for an unnaturally large scattering length $(|a| \gg 1 / \Lambda)$, the scattering-length approximation for a meson-nucleon $T$-matrix is not justified, and the inclusion of the unitarity, recoil, and/or range corrections becomes necessary. Once this is done the pole may be shifted towards the origin, and so move into a region outside the domain of applicability of the EFT, or it may even disappear completely. However, additional investigations are necessary in order to confirm this conjecture.

This scenario might be expected to lead to difficulties for kaon-nucleus scattering, since $a_{K N}$ is negative and large enough in certain channels that the MSS may have a pole in the physical region. However, for an isovector-dominated mesonnucleon amplitude a pole never appears in the resummed series. In addition, in non-relativistic EFT, which is used for $K d$ scattering, the pole could appear only quite close to the origin since $\Lambda|a|$ is only slightly larger than 1 . Therefore no enhanced counterterms are necessary in order to render the MSS for kaon-nucleus scattering sensible.

Similar resummations of the MSS graphs which appear in the $N N$ potential are also possible. In that case the potential does develop a pole at a finite radius $r$. This implies that the $\chi \mathrm{PT}$ expansion for $V_{N N}$ has already broken down once the pole appears. This suggests a limitation of $r>1 \mathrm{fm}$ for the successful application of $\chi \mathrm{PT}$ to $V_{N N}(r)$, although this limit could be lowered if dynamical Delta degrees of freedom are included in the EFT.

\section{Acknowledgments}

This research was supported by the DFG (SFB/TR 16, "Subnuclear Structure of Matter"), DFG-RFBR grant (436 RUS 
113/991/0-1), the Bonn-Cologne Graduate School of Physics and Astronomy, the DAAD, the project "Study of Strongly Interacting Matter" (HadronPhysics3) under the 7th Framework Programme of the EU, the European Research Council (ERC2010-StG 259218 NuclearEFT), and the US Department of Energy (Office of Nuclear Physics, under contract No. DE-FG0293ER40756 with Ohio University).

\section{References}

1. L. L. Foldy, Phys. Rev. 67 (1945) 107.

2. K. A. Brueckner, Phys. Rev. 89 (1953) 834; Phys. Rev. 90 (1953) 715 .

3. V. M. Kolybasov and A. E. Kudryavtsev, Nucl. Phys. B 41 (1972) 510 .

4. S. Weinberg, Phys. Lett. B $295 \quad$ (1992) 114 [arXiv:hep-ph/9209257].

5. S. R. Beane, V. Bernard, T. S. H. Lee and U.-G. Meißner, Phys. Rev. C 57 (1998) 424 |arXiv:nucl-th/9708035|.

6. S. R. Beane, V. Bernard, E. Epelbaum, U.-G. Meißner and D. R. Phillips, Nucl. Phys. A 720 (2003) 399 [arXiv:hep-ph/0206219].

7. V. Baru, C. Hanhart, A. E. Kudryavtsev and U.-G. Meißner, Phys. Lett. B 589 (2004) 118 |arXiv:nucl-th/0402027|.

8. V. Lensky, V. Baru, J. Haidenbauer, C. Hanhart, A. E. Kudryavtsev and U.-G. Meißner, Phys. Lett. B 648 (2007) 46 [arXiv:nucl-th/0608042].

9. V. Baru, J. Haidenbauer, C. Hanhart, A. E. Kudryavtsev, V. Lensky and U.-G. Meißner, Phys. Lett. B 659 (2008) 184 [arXiv:0706.4023 [nucl-th]].

10. S. Liebig, V. Baru, F. Ballout, C. Hanhart and A. Nogga, Eur. Phys. J. A 47 (2011) 69 arXiv:1003.3826 [nucl-th]].

11. V. Baru, C. Hanhart, M. Hoferichter, B. Kubis, A. Nogga and D. R. Phillips, Phys. Lett. B 694 (2011) 473 [arXiv:1003.4444 [nucl-th]].

12. V. Baru, C. Hanhart, M. Hoferichter, B. Kubis, A. Nogga and D. R. Phillips, Nucl. Phys. A 872 (2011) 69 arXiv:1107.5509 [nucl-th]].

13. D. Gotta et al., Lect. Notes Phys. 745 (2008) 165.

14. T. Strauch et al., Eur. Phys. J. A 47 (2011) 88 [arXiv:1011.2415 [nucl-ex]].

15. S. S. Kamalov, E. Oset and A. Ramos, Nucl. Phys. A 690 (2001) 494 [arXiv:nucl-th/0010054].
16. U.-G. Meißner, U. Raha and A. Rusetsky, Eur. Phys. J. C 47 (2006) 473 [arXiv:nucl-th/0603029].

17. V. Baru, E. Epelbaum and A. Rusetsky, Eur. Phys. J. A 42 (2009) 111 [arXiv:0905.4249 [nucl-th]].

18. A. W. Thomas and A. S. Rinat, Phys. Rev. C 20 (1979) 216.

19. Y. Avishai and T. Mizutani, Phys. Rev. C 27 (1983) 312

20. I. R. Afnan and B. Blankleider, Phys. Rev. C 32 (1985) 2006.

21. D. R. Phillips and I. R. Afnan, Annals Phys. 247 (1996) 19 [arXiv:nucl-th/9502040].

22. A. N. Kvinikhidze and B. Blankleider, Nucl. Phys. A 574 (1994) 788 arXiv:nucl-th/9402010].

23. E. Epelbaum and J. Gegelia, Eur. Phys. J. A 41 (2009) 341 [arXiv:0906.3822 [nucl-th]].

24. E. Epelbaum, W. Glöckle and U.-G. Meißner, Nucl. Phys. A 747 (2005) 362 [arXiv:nucl-th/0405048].

25. G. P. Lepage, arXiv:nucl-th/9706029.

26. M. C. Birse, Phys. Rev. C 74 (2006) 014003 arXiv:nucl-th/0507077.

27. A. Nogga, R. G. E. Timmermans and U. van Kolck, Phys. Rev. C 72 (2005) 054006 [arXiv:nucl-th/0506005].

28. M. Pavon Valderrama and E. R. Arriola, Phys. Rev. C 74 (2006) 054001 [arXiv:nucl-th/0506047].

29. E. Epelbaum and U.-G. Meißner, arXiv:nucl-th/0609037

30. C. Ordonez, L. Ray and U. van Kolck, Phys. Rev. C 53 (1996) 2086 [hep-ph/9511380].

31. N. Kaiser, R. Brockmann and W. Weise, Nucl. Phys. A 625 (1997) 758 |arXiv:nucl-th/9706045].

32. V. Bernard, N. Kaiser and U.-G. Meißner, Nucl. Phys. A 615 (1997) 483 [arXiv:hep-ph/9611253.

33. H. Krebs, E. Epelbaum and U.-G. Meißner, Eur. Phys. J. A 32 (2007) 127 [arXiv:nucl-th/0703087].

34. M. P. Valderrama, Phys. Rev. C 83 (2011) 024003 [arXiv:0912.0699 [nucl-th]].

35. M. C. Birse, Eur. Phys. J. A 46 (2010) 231 arXiv:1007.0540 [nucl-th]].

36. K. L. Ipson, K. Helmke and M. C. Birse, Phys. Rev. C 83 (2011) 017001 [arXiv:1009.0686 [nucl-th]].

37. M. P. Valderrama, Phys. Rev. C 84 (2011) 064002 arXiv:1108.0872 [nucl-th]].

38. N. Kaiser, S. Gerstendorfer and W. Weise, Nucl. Phys. A 637 (1998) 395 [arXiv:nucl-th/9802071.

39. S. R. Beane and M. J. Savage, Nucl. Phys. A 694 (2001) 511 [nucl-th/0011067]. 